\title{
Faraday cage screening reveals intrinsic aspects of the van der Waals attraction
}

\author{
Musen Li (李木森) ${ }^{a, b}$, Jeffrey R. Reimers ${ }^{a, b, c, 1}$ ， John F. Dobson ${ }^{d, e}$, and Tim Gould ${ }^{d, e, 1}$ \\ anternational Centre for Quantum and Molecular Structures, Shanghai University, Shanghai 200444, China; ${ }^{\mathrm{b}}$ Department of Physics, Shanghai University, \\ Shanghai 200444, China; 'School of Mathematical and Physical Sciences, University of Technology Sydney, Ultimo, NSW 2007, Australia; dSchool of Natural \\ Sciences, Griffith University, Nathan, QLD 4111, Australia; and ${ }^{\mathrm{e}}$ Queensland Micro- and Nanotechnology Centre, Griffith University, Nathan, QLD 4111, \\ Australia
}

Edited by John P. Perdew, Temple University, Philadelphia, PA, and approved September 21, 2018 (received for review July 5, 2018 )

General properties of the recently observed screening of the van der Waals (vdW) attraction between a silica substrate and silica tip by insertion of graphene are predicted using basic theory and first-principles calculations. Results are then focused on possible practical applications, as well as an understanding of the nature of vdW attraction, considering recent discoveries showing it competing against covalent and ionic bonding. The traditional view of the vdW attraction as arising from pairwiseadditive London dispersion forces is considered using Grimme's "D3" method, comparing results to those from Tkatchenko's more general many-body dispersion (MBD) approach, all interpreted in terms of Dobson's general dispersion framework. Encompassing the experimental results, MBD screening of the vdW force between two silica bilayers is shown to scale up to medium separations as $1.25 d_{\mathrm{e}} / d$, where $d$ is the bilayer separation and $d_{e}$ is its equilibrium value, depicting antiscreening approaching and inside $d_{\mathrm{e}}$. Means of unifying this correlation effect with those included in modern density functionals are urgently required.

van der Waals screening | Faraday cage | covalent bonding | many-body dispersion | Dobson B

M aterials in zero, one, two, and three dimensions of relevance to conceived future fabrication and electronics technologies are often held together by the van der Waals (vdW) dispersion force (1). Methods of measuring and first-principles simulations of the free energies of formation of such systems are becoming available (2). Often the critical issues involve situations in which the forces holding systems together become nonadditive, that is, the interaction between two parts of a system is modulated by the presence of nearby matter, with dispersion and other aspects all contributing (3, 4). At short distances typical of chemical bonding, it is now being recognized that dispersion forces can sometimes compete with traditional chemical covalent and ionic bonding forces to control outcomes by a number of means (5-13). Related parallel work demonstrates how ionic forces can control typical scenarios associated with dispersion (14), as well as scenarios in which general solvent effects including dispersion control structure $(15,16)$. Alternatively, at long distances, the Casimir dispersion effect becomes critical (17-19), as well as other exotic phenomena associated with the wavelike nature of charge polarization in nanoscale objects (4). While answers to each of the issues raised can be formed in isolation, a generally useful understanding of the vdW force remains elusive. Indeed, how different computational methods perceive dispersion forces at long and short distances have been found to be uncorrelated, raising fundamental questions concerning the nature of the force (12). What happens to long-range phenomena at vdW separations and then as chemical bond distances are reached will form a key part of future understanding.

To initiate discussion, we consider the extremely nonadditive $\mathrm{vdW}$ interactions observed by Tsoi et al. (20) in systems involving a silica substrate, a silica atomic force microscope (AFM) tip, and an intervening conducting graphene layer. The remarkable result from this work is that a large interobject dispersion force was switched off by the insertion of graphene in between the objects. Here, the origin and basic properties of this effect are elucidated using first-principles computational methods applied to a model 2D system. Discussion is considered using the framework for understanding vdW phenomena developed recently by Dobson (21).

Studies of 2D materials are currently very prevalent, with firstprinciple simulations providing powerful tools to facilitate understanding $(11,22-25)$. While dispersion interactions are critical for determining the structure and properties of such systems, the most commonly used method applied for first-principles materials simulations, density functional theory (DFT) using a conventional generalized-gradient approximation (GGA), improperly treats its contribution. As a result, a wide range of empirical correction schemes are commonly added to GGA calculations so as to produce a realistic description of the critical interactions $(24,25)$. The vdW dispersion interactions described by these schemes typically involve sums over interatomic interactions, each described by the London force (21, 26-28), with only small corrections. Related variants include replacing the atomic sums by electron-density integrals. In either case, these approaches are intrinsically pairwise additive, meaning that adding more atoms to the system just systematically increases the net dispersion interaction.

\section{Significance}

How the van der Waals dispersion interaction relates to chemical electron-correlation effects presents a critical challenge to density functional theory development. Here, recently observed screening of the dispersion force between two insulating objects caused by the insertion of an intermediary graphene layer is explained in terms of Dobson's general description of dispersion. This then provides a much-needed handle concerning how density functional approaches relate such long-range dispersion interactions to the subtleties of covalent bonding. Screening at intermediate distances appears to change the London expression from $r^{-6}$ to $r^{-7}$, an effect that becomes antiscreening (dispersion enhancement) at distances shorter than van der Waals contact. This provides basic insight into modern revelations that dispersion forces can outcompete covalent forces to control chemical structure.

Author contributions: J.R.R., J.F.D., and T.G. designed research; M.L. performed research; M.L., J.R.R., J.F.D., and T.G. analyzed data; and M.L., J.R.R., and T.G. wrote the paper. The authors declare no conflict of interest.

This article is a PNAS Direct Submission.

This open access article is distributed under Creative Commons Attribution-NonCommercialNoDerivatives License 4.0 (CC BY-NC-ND).

${ }^{1}$ To whom correspondence may be addressed. Email: jeffrey.reimers@uts.edu.au or t.gould@griffith.edu.au.

Published online October 16, 2018. 
We select two widely applied types of methods to investigate the Tsoi et al. (20) experiment and its wider implications. First, we use pairwise-additive approaches based on "D3"-type schemes of Grimme that have achieved wide-ranging success $(2,29-33)$, particularly when applied to understand chemical vdW structures and energetics at equilibrium separations. These methods are also being found useful for understanding dispersion contributions down to chemical bond-length scales $(10,12$, $31,34)$. Second, we apply the more general many-body dispersion (MBD) approaches of Tkatchenko and others (4, 24, 35-45) that have also been shown to be widely successful, especially when large objects are involved at both equilibrium and larger separations. Of general interest is how different methods treat the vdW force during small excursions from equilibrium (46). More significantly, the observation that the vdW force can be switched off severely challenges the traditional pairwise-additive point of view and hence provides an external reference frame for this discussion. Here, combining the results from the different computational methods reveals that the switching off of the vdW interaction is an effect somewhat analogous to the "Faraday cage" effect that is well known throughout physics and engineering. The model system used for these calculations is shown in Fig. 1 and contains two bilayer silicon sheets separated by vacuum from an interposed graphene layer.

Dobson's (21) framework for understanding the dispersion force conceptually links traditional ideas concerning pairwise additivity to "top-down" Lifshitz theory in which screening emerges as a natural consequence of the quantum fluctuations that facilitate the dispersion interaction $(47,48)$. In the traditional view, each interatomic interaction is specified by the London contribution (49), which scales as $r^{-6}$, where $r$ is the interatomic distance, with only small corrections. The origin of the basic interaction is the polarization of one atom by spontaneous quantum dipole fluctuations occurring inside another.

At the simplest level, all atoms of the same type could be treated as having the same vdW attractions (the gas-phase values) independent of chemical environment. Dobson classified corrections to this approach into three types (21). The first type, here called "Dobson A," focuses on the quantal effect of the
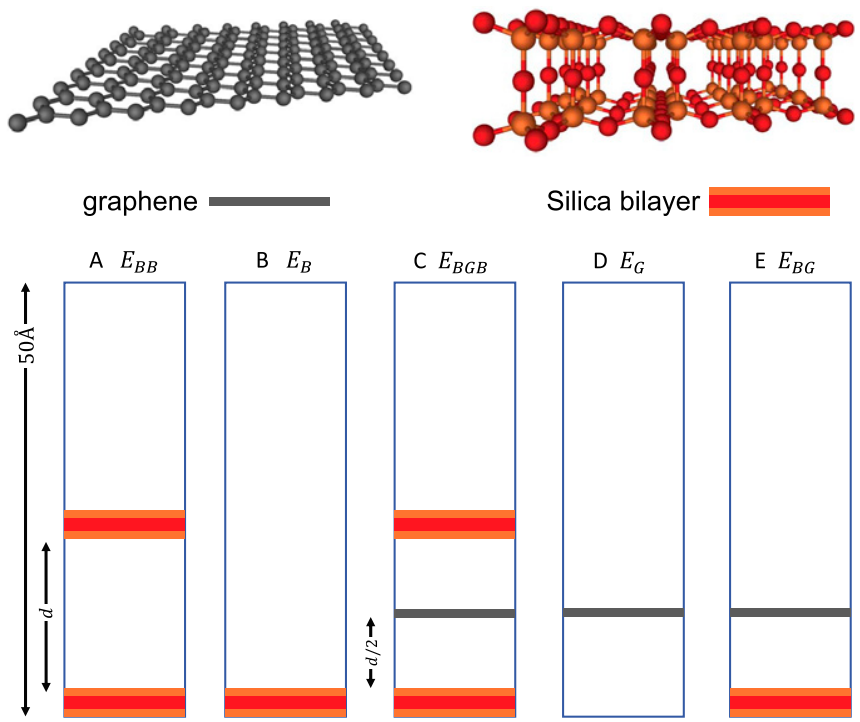

Silica bilayer
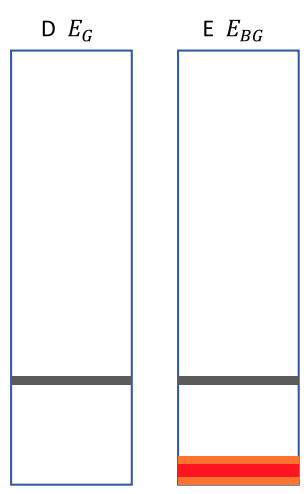

Fig. 1. Geometries used for determining the screening of the vdW interaction between two silica bilayers induced by inserting an intermediary graphene sheet. $(A)$ Two silica bilayers, $(B)$ just one silica bilayer, $(C)$ full system with graphene inserted midway between the two silica bilayers, $(D)$ graphene only, and $(E)$ graphene plus just a single silica bilayer. local chemical environment, insofar as it modifies the polarizability of each atom (e.g., via orbital compression) and hence modifies its vdW interaction with other atoms. Just bringing two chemically noninteracting atoms close together induces a Dobson-A effect as the polarizability of one atom will change owing to the presence of the other. Most modern computational methods treat such terms to high accuracy, sometimes through empirical parameterization and sometimes through explicit environmental modeling.

In the second type, here called "Dobson B," the fluctuating electric fields that mediate the vdW interaction between a pair of atoms are disrupted (screened or antiscreened) by the sympathetic induced fields caused by polarization of other atoms. This produces long-ranged "many-body" (many-atom) vdW effects $(21,27)$.

A third type of correction, called "Dobson C," involves longranged fluctuating charge transfer occurring on a length scale larger than the size of an atom. This becomes particularly significant for long-ranged interactions between low-dimensional metallic conductors $(26,50,51)$, but, in undoped graphene at $T=0 \mathrm{~K}$, it is less important (22). It leads on (21) to the Casimir effect (17-19), something that can become very important in large systems (52).

Hence, in the context of the Tsoi et al. (20) experiment, Dobson-B effects take on a central focus: $(i)$ What are they? (ii) how reliably do modern computational methods treat them? (iii) can they account for the observed screening of the silica-silica dispersion attraction by graphene? (iv) how do they influence critical equilibrium properties of vdW heterostructures? ( $v$ ) how do they influence the way that dispersion forces are perceived at shorter distances of the order of chemical bond lengths? (vi) how do they behave asymptotically at long range and at very short range in the unified-atom limit? and (vii) can they be manipulated to make new functional materials and devices?

\section{Results}

Nature of the Dobson-B Screening Effect and Its Relationship to a Faraday Cage. Fig. 2 illustrates the significance of type B (21) effects in the situation that we will model here, namely, a monolayer graphene sheet midway between two well-separated silica sheets. The graphene sheet will turn out to reduce the dispersion interaction between the two $\mathrm{SiO}_{2}$ sheets in a process analogous to the operation of a Faraday cage. Faraday caging arises within classical electrostatics, preventing an external electric field from penetrating through an infinite conducting sheet or a continuous metallic surface or "cage." Typical ramifications of this effect include the blocking of radio waves by a steel-framed bridge and the reduction of mobile-phone signals by typically a million-fold by placing a MRI machine inside a room with continuous metallic walls. Polarization of the atoms within the metal by the external field cooperate across the whole extent of the metal to prevent penetration of the electric and magnetic fields. The effect applies independent of the source of the electric field, for example, permanent charges, electromagnetic radiation, or, as considered here, the spontaneous quantum dipole fluctuations involved in vdW attractions. Hence, screening induced by the Dobson-B effect can be thought of as a generalization of the abovementioned traditional notions of Faraday caging. However, vdW screening involves electronic fluctuations happening on the attosecond-femtosecond timescales, whereas Faraday caging formally involves responses at zero frequency, with microwave frequencies being so low in comparison that they are considered "zero frequency" in this context. Our application of the expression "Faraday cage" herein is also a little loose as its meaning is usually associated with continuous metallic conductors, whereas here we discuss atomistic graphene sheets. Certainly, the shielding of the fluctuating fields by an undoped, cold graphene sheet should be less complete than the screening of 


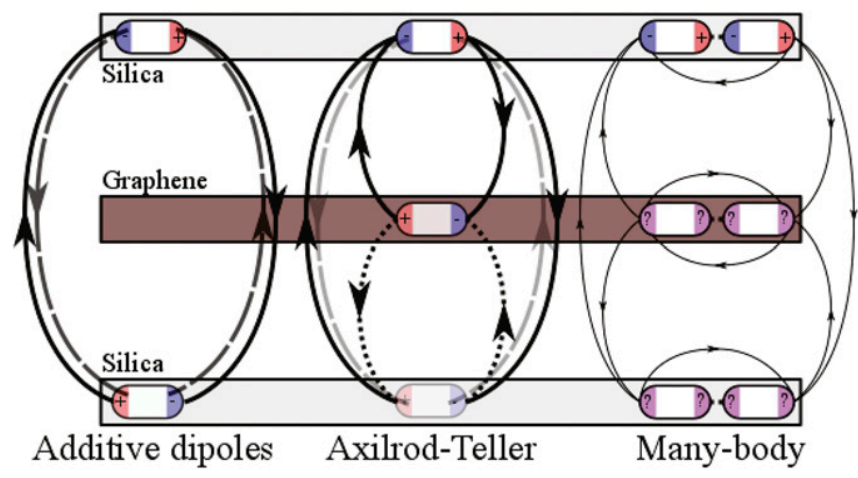

Fig. 2. MBD interactions. Electric field lines (solid) produced by a short-lived spontaneous dipole on a top-layer atom induce polarization of other atoms: These atoms therefore produce reaction fields (dashed or dotted). Weaker fields and dipoles are here shown as fainter. See the text for a detailed analysis of the diagrams. A full many-body treatment was required for the Faraday cage reported here.

static fields by a true metal sheet, but it is still substantial, as will be demonstrated later. With these qualifications, the Faraday cage concept captures the essential essence of an important aspect of Dobson B, providing simple understanding to the Tsoi et al. (20) experiment.

As depicted in Fig. 2, Left, pairwise dispersion theories that neglect Dobson- $\mathrm{B}$ effects give the direct $\mathrm{SiO}_{2}-\mathrm{SiO}_{2}$ dispersion energy via interaction of the original top dipole with the dashed reaction field generated by the induced bottom dipole (53). This process misses simultaneous interactions involving both silica bilayers and graphene, and so cannot describe Faraday caging. The simplest way to include such interactions is through the three-atom AxilrodTeller interaction (also known as the Axilrod-Teller-Muto interaction) terms (middle diagram in Fig. 2). These arise because the solid field lines from the top dipole also induce dipoles in the graphene layer. A graphene dipole then produces the dotted field line, which tends to cancel the solid direct field line, at the position of the atom in the lower $\mathrm{SiO}_{2}$ layer. This reduces the dipole induced on the bottom atom, and thereby reduces the dashed reaction field (fainter dashed lines). This in turn reduces the vdW interaction between atoms in the top and bottom layers. It is genuinely a threeatom effect, additional to the pairwise interactions of all species. The middle atom has "screened" the pair interaction between top and bottom atoms, yielding a partial Faraday cage effect.

Another way to view the three-atom Axilrod-Teller effect is the following. The polarizability of atoms in the combined subsystem (graphene plus bottom $\mathrm{SiO}_{2}$ layer) differs from the sum of the individual polarizabilities owing to the Coulomb interaction that occurs between the bottom two layers. This change of polarizability then affects the vdW interaction between atoms in the top $\mathrm{Si}$ layer and those in the combined (graphene plus lower $\mathrm{SiO}_{2}$ ) system.

However, this Axilrod-Teller term is only the simplest level of the Dobson-B electrostatic screening phenomenon as unique contributions also appear when four atoms are simultaneously considered, etc. Many-body terms (Fig. 2, Right) lead to a more complete description of Faraday cage screening. Here, any atom can screen (or enhance, "antiscreen") the vdW interaction between any others, in accordance with the global geometry of the system. In this many-body case, for simplicity we do not distinguish initiating dipoles and fields from induced ones, showing all field lines as thin solid lines in the right-hand diagram of Fig. 2.

Inclusion of Dobson-B Effects in Electronic Structure Calculations of Molecules and Materials. Among ab initio computational approaches, the simplest that includes dispersion is second-order
Møller-Plesset theory (54) (MP2). This includes dispersion up to Dobson A only. Higher-order methods like coupled-cluster singles and doubles theory (CCSD) (55) and the random-phase approximation (RPA) (21) include also elements of both Dobson $\mathrm{B}$ and Dobson $\mathrm{C}$.

In DFT approaches, most treatments of dispersion, including double-hybrid functionals, are based intrinsically on the assumption of pairwise additivity of the dispersion energy $(21,25)$, thus excluding explicit Dobson-B effects. These include the D3 family [except D3(ABC), which has some Axilrod-Teller terms] $(29,30)$, the XDM method (56), and many older approaches (57-60). All practical computational methods involve many assumed equations and their parameters, with some methods (like D3) being fully empirical with many parameters, while others are semiempirical, specifying equations that automatically generate required properties. Some methods may go beyond pure pairwise additivity by various means; D3(ABC) explicitly includes some three-body contributions, while most other methods include an uncontrolled amount of Dobson-B contributions into the Dobson-A terms (25) by modifying the pairwise-additive energies based only on the immediate chemical environment. D3 appears as an exemplary method demonstrating great success when screening and antiscreening effects can be treated empirically $(2,29-33)$, while D3(ABC) appears as the first step in a perturbative improvement strategy.

Going beyond these levels of treatment, of the efficient, semiempirical DFT+dispersion approaches, only the MBD method of Tkatchenko et al. $(35,41)$ and its descendants $(24,42)$ explicitly include Dobson-B corrections to all orders. It does so in a way that depends on global geometries and so includes Faraday screening and other many-body effects. This approach is known to be very successful in treating screening and antiscreening interactions at short range, that is, separations at and just beyond vdW contact (4, $24,35-45)$. It is inappropriate for small-gap systems at very long ranges (21), but the separation used in the Tsoi et al. (20) experiment is just $20 \mathrm{~nm}$, an intermediate distance at which MBD would still be expected to apply.

Very recently, a complementary interpretation of the Tsoi et al. (20) experiment was presented using a specialist method embodying Dobson B. In contrast to MBD-based approaches, this new approach was developed only to treat only very longrange interactions (17) and is inappropriate at vdW contact distances and thereabouts. It uses a model dielectric for graphene that does not involve the necessary cutoff to the intraband "Dirac cone" transitions and also neglects important interband transitions. Consequently, it is applicable only at very large distances (61).

An alternative method that encompasses the effects included in both MBD $(35,41)$ and this new work $(17)$, as well as Dobson $\mathrm{C}$ and other effects, is RPA applied using DFT orbitals (62) ("dRPA"). This includes, at a realistic level, all dispersive effects operating over all length scales $(53,63)$. However, at the moment it is not computationally feasible to apply it to the problem at hand; nevertheless, efficient RPA methods are emerging (6468 ), presenting a variety of possible future options.

Hence, at the moment, only MBD and its descendants appear as feasible approaches for capturing screening effects in the complex chemical environment of the silica-graphene system from the recognized important regime of close contact up to the separations of primary interest herein. Demonstration that this approach can account for a large reduction of the vdW attraction between silica bilayers on insertion of graphene, whereas methods that do not fully include Dobson B cannot, would present a result indicative that Faraday cage screening is responsible for the effect seen in the Tsoi et al. (20) experiments, connecting this effect to problems of great interest in biochemistry, chemistry, and condensed-matter physics. 
Evidence for Faraday Cage Screening by Graphene. The bilayer silica (20) used in our computational model constitutes a 2D layered material that has similar properties to usual 3D silica. However, it provides a test system small enough to allow calculations to very high precision $(10 \mu \mathrm{eV})$, as is required for the evaluation of the dispersion force between bilayers over a large range in separations. A variety of specialized techniques are required to converge calculations to this precision, as detailed in Materials and Methods. To further enhance precision, the geometries of the silica bilayers and the graphene sheet were frozen at their individually optimized values. This is a poor approximation at and inside $\mathrm{vdW}$ contact, but is very adequate in the critical intermediate-range region. Similarly, we choose a commensurate lattice with lattice parameter of $5.18 \AA$ for silica and graphene. This preserves key qualitative properties of both materials, such as band structures, but ensures the cell size is reasonable; key results are insensitive to the chosen lattice parameter.

To determine the dispersion interaction between two silica bilayers and the effect of intervening graphene on it, calculations are performed for five systems, as described in Fig. 1. Fig. $1 A$ shows two silica bilayers separated by interfacial distance $d$; its energy is labeled $E_{B B}$. To get the unscreened dispersion energy $\Delta E_{u}$ at this distance, the energy of the corresponding isolated single bilayers, $E_{B}$, obtained from the structure shown in Fig. $1 B$ is subtracted, yielding the following:

$$
\Delta E_{u}=E_{B B}-2 E_{B} .
$$

To get the screened dispersion interaction $\Delta E_{s}$, we insert a graphene layer in the center between two silica bilayers, as shown in Fig. $1 C$. The energy of this structure is labeled $E_{B G B}$, from which must be subtracted the energies of each component and the energies of each silica-graphene dispersion interaction. To do this, we evaluate the energy of an isolated graphene sheet $E_{G}$ (Fig. $1 D$ ) and the energy of the bilayer-graphene interaction at distances $d / 2, E_{B G}$ (Fig. $1 E$ ), yielding the following:

$$
\Delta E_{s}=E_{B G B}-2 E_{B G}+E_{G} .
$$

Of particular interest is the ratio $\Delta E_{s} / \Delta E_{u}$ specifying the reduction of the intersilica-bilayer dispersion force induced by the insertion of graphene. In addition, the total binding energy between the three layers is given by the following:

$$
\Delta E=E_{B G B}-E_{G}-2 E_{B} .
$$

The D3 and MBD computational approaches both have multiple variants. For D3, we select the methods knows as "D3(BJ)" and "D3(BJ,ABC)," both involving Becke-Johnson damping, and where $\mathrm{ABC}$ indicates that some three-body Axilrod-Teller terms are included $(29,69)$. For MBD $(41,70)$, we employ the fractional ionic (FI) method (43), which incorporates a superior polarizability model for oxides and a more physical treatment of many-body screening effects compared with the original method. Full details of the computational methods used are provided in Materials and Methods (71-75).

Fig. $3 A$ shows the total binding energy $\Delta E$ as a function of the interlayer spacing $d$, revealing that the predicted equilibrium separations are $d_{e}=6.65 \AA$ both for MBD-FI and D3(BJ,ABC) or else $6.50 \AA$ for $\mathrm{D} 3(\mathrm{BJ})$, all in the range reported by Włodarczyk et al. (76). Next, Fig. $3 B$ shows the unscreened vdW dispersion interaction $\Delta E_{u}$. All three methods considered give similar results for small $d$, with $\mathrm{D} 3(\mathrm{BJ})$ predicting $\sim 30 \%$ larger values than the other methods at equilibrium and hence also $\sim 30 \%$ larger binding $\Delta E$. While the magnitude of the additional binding strength predicted by $\mathrm{D} 3(\mathrm{BJ})$ is significant, the overall similarity of the results reflects the observation that all of these
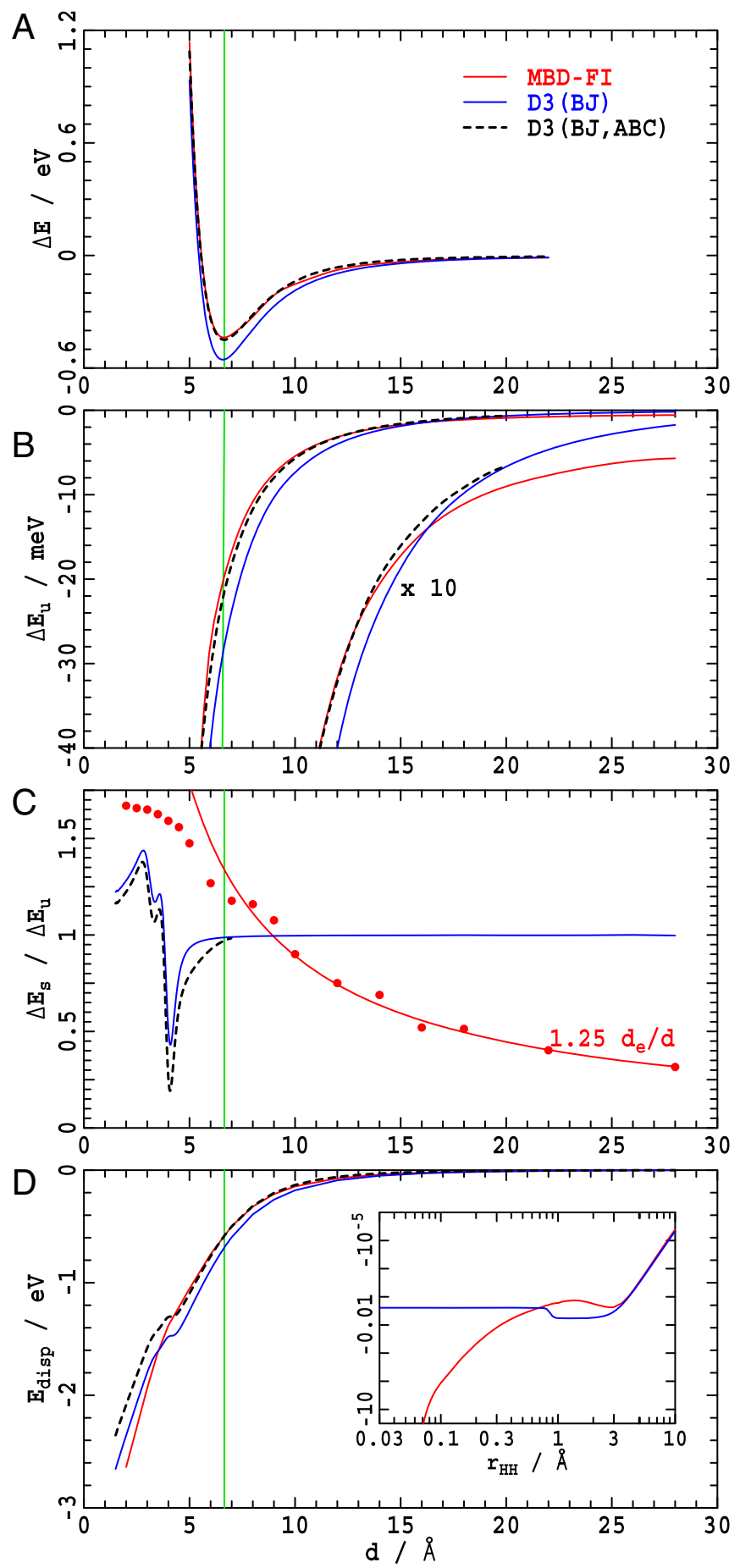

Fig. 3. Screening at work. $(A)$ The total interaction energy $\Delta E$ at interlayer distance $d$ (Fig. 1). (B) The contributions to this arising from the unscreened silica-silica vdW energies $\Delta E_{u}$ obtained without an intervening graphene. (C) The screening ratio $\Delta E_{s} / \Delta E_{u}$ obtained after insertion of graphene. $(D)$ The total dispersion energy for the BGB system, with the Inset showing that for the $\mathrm{H}_{2}$ molecule on a log-log scale. The green line indicates the equilibrium separation of $d_{\mathrm{e}}=6.65 \AA$ as calculated using MBD-FI and D3(BJ,ABC); D3(BJ) gives $6.60 \AA$ A instead.

methods can describe most systems in vdW contact $(2,24,25$, 77). Most significantly, the results from the very different approaches $\mathrm{D} 3(\mathrm{BJ}, \mathrm{ABC}$ ) and MBD-FI shown in Fig. $3 A$ (for $\Delta E$ ) and Fig. $3 B$ (for its small component $\Delta E_{u}$ ) are mostly in excellent agreement. 
However, how the various approaches treat the small bilayerbilayer contribution to the total energy upon insertion of graphene, a quantity analogous to that measured in the Tsoi et al. (20) experiments, is highlighted in Fig. $3 C$, where the ratio $\Delta E_{s} / \Delta E_{u}$ is shown. A purely pairwise-additive method would yield $\Delta E_{s} / \Delta E_{u}=1$ at all geometries, and the results for both D3 variants are indeed very close to this value at vdW contact and beyond. Small deviations occur through explicit inclusion of three-body corrections and the environment dependence of the parameters used in the method and, for larger distances, through rounding errors. In striking contrast, the MBD-FI ratio shown in Fig. $3 C$ are fitted to yield the following:

$$
\frac{\Delta E_{s}}{\Delta E_{u}}=1.25 \frac{d_{e}}{d}
$$

reducing toward zero with a $d^{-1}$ dependence. The screening is half the equilibrium value at twice the equilibrium bond length, but the value of 1.25 at equilibrium indicates that antiscreening (factor $>1$ ) is occurring at this critical geometry. One feature relevant here is that perfect Faraday cage screening only happens for continuous metallic conductors, and at short distances close to interatomic spacings, electric fields can penetrate through materials. Furthermore, the electron correlation effects manifested as the dispersion interaction must smoothly connect with the strong electron correlation effects manifested at short range within the GGA approximation, making the atomic nature of matter an unlikely cause of the antiscreening. In any case, a key feature of interest is that, at equilibrium distances, the net effect of Dobson-B terms becomes small (here, a $25 \%$ correction, with also a kink in the curve making the derivative small at this critical geometry), allowing sensibly parameterized pairwise-additive models to depict realistic many important features $(2,31,32,78)$.

However, the screening increases rapidly as the layers separate, reducing the unscreened vdW force to half at about twice the equilibrium separation. In this way, Dobson-B effects can become important even at or close to equilibrium separations, and approaches like MBD have been shown to have significant advantages in a range of applications $(4,24,35,37-43)$.

At moderately long range, extrapolation the MBD-FI results using Eq. 3 predicts, at the separation of $d=20 \mathrm{~nm}$ as used in the Tsoi et al. (20) experiments, that the screening ratio $\Delta E_{s} / \Delta E_{u}$ should be $4.4 \%$, close to the observed value of $5 \%$. The calculations thus provide an explanation for the observation.

Anticipated Properties at Long Range. The MBD-FI calculations and the Tsoi et al. (20) experiments consider screening in the 0.2to 3-nm range and at $20 \mathrm{~nm}$, respectively. Anticipated properties asymptotically at long range are also worthy of consideration. As an example of Faraday cage screening, we note that a 2D continuous metallic layer will completely reflect long-wavelength static $(\omega=0)$ electric fields, but even for a metal, dynamic (high-frequency) fields are not fully reflected. The dispersion interaction depends on the exchange of such dynamic (high $\omega$ ) electric fields. An undoped, zero-temperature graphene sheet is a good electrical conductor at zero frequency, but graphene is not a metal in the usual sense, having a zero electronic density of states at the Fermi energy. Correspondingly, it is known (68) that such a single undoped graphene layer, at $T=0 \mathrm{~K}$ as modeled herein, in contrast to a 2D metal, reflects most but not all of any long-wavelength static field, and this refection coefficient will be reduced at the finite frequencies of interest for the dispersion interaction. It is therefore not obvious how complete the Faraday caging effect will become for an undoped zero-temperature graphene sheet. The fitted $d^{-1}$ dependence (Eq. 3) found at short to intermediate distances may therefore in reality be replaced asymptotically by a constant minimum value. This is consistent with recent long-range calculations (17).

How the MBD method is expected to perform at very long range is also of interest. Rather than manifesting this anticipated theoretical limit, the MBD approach actually treats graphene as an insulator. Hence, asymptotically, MBD is expected to produce no screening at all (if it could be carried out numerically at asymptotic separations), and, like D3, would yield $\Delta E_{s} / \Delta E_{u}=1$.

Insight into the vdW Force and Its Means of Computation. Most chemical discussion of the vdW force considers it as an example of the effects of electron correlation operating at distances above two to three times chemical bond lengths. Table 1 summarizes understanding of its properties as well as more "chemical" properties associated with smaller length scales. The simplest approach for describing electronic structure, Hartree-Fock theory $(\mathrm{HF})$, treats electrons as effective noninteracting particles, ignoring all electron correlation. It is qualitatively descriptive of covalent and ionic bonding but omits the subtle features often responsible for quantitative analysis of chemical reaction property differences and, accordingly, does not include any vdW effects.

Its ab initio improvement, MP2 theory, includes both shortrange and long-range correlation in a pairwise fashion, greatly improving chemical reaction properties as well as describing vdW bonding, but Dobson-B effects like screening and antiscreening are not included. The excluded effects are well known to influence chemical bonding at short range, and here we see that they are also can be profound at long range. This shows that Dobson-B effects are influential over all length scales. At the next level of ab initio improvement to HF theory, methods like CCSD and RPA (approximately) include all effects, providing

Table 1. Overview of the effects of electron correlation

\begin{tabular}{|c|c|c|c|c|}
\hline Example method & Correlation type & Effect at chemical bond lengths & $\begin{array}{l}\text { Effect at near } \\
\text { vdW separations }\end{array}$ & $\begin{array}{l}\text { Long-range Dobson-B effects } \\
\text { like screening and antiscreening }\end{array}$ \\
\hline HF & None & $\begin{array}{l}\text { Qualitatively descriptive of covalent and ionic } \\
\text { bonds but poor quantitative accuracy }\end{array}$ & Not included & Not included \\
\hline $\mathrm{HF}+\mathrm{D} 3$ & None & Akin to that from GGAs & Mostly quantitative & Not included \\
\hline MP2 & Fully pairwise & Quantitatively useful & Descriptive & Not included \\
\hline CCSD, RPA & Fully many-body & Quantitative & Quantitative & Quantitative \\
\hline GGA & Local many-body & Quantitatively useful & Improperly described & Improperly described \\
\hline GGA+D3 & $\begin{array}{l}\text { Local many-body + } \\
\text { pairwise vdW }\end{array}$ & Quantitativity improved by D3 & Mostly quantitative & Not included \\
\hline GGA+MBD & $\begin{array}{l}\text { Local many-body + } \\
\text { many-body vdW }\end{array}$ & Quantitativity reduced by MBD & Quantitative & $\begin{array}{l}\text { Adequate at short to } \\
\text { intermediate range* }\end{array}$ \\
\hline Ref. 17 & Long-range only & Inappropriate & Inappropriate & Designed for long range \\
\hline
\end{tabular}

*Except for low-dimensional conductors for which Dobson C becomes critical, as may also be the related Casimir effect. 
bonding descriptions to chemical accuracy as well as including screening and antiscreening (21). While these methods do not directly provide insight in terms of intuitive descriptors like "chemical bonding" as distinct from "vdW bonding," they embody a smooth link between these concepts.

DFT approaches like GGAs with vdW corrections are in much more widespread use owing to their computational efficiency and their ready applicability to both periodic systems and systems with small or no band gap, but the great challenge for the present concerns how to link together their disparate descriptions of chemical bonding and vdW bonding. GGAs provide an exact description of electron correlation in a free-electron gas over all length scales and so in principle fully include dispersion. However, they do so in a way that yields very poor results in any atomic system, defining the core problem needing to be solved (Table 1). Of note is that they embody a fully many-body description of short-range electron correlation that typically provides a better description of chemical properties than does a purely pairwise method like MP2.

So the situation is that many-body effects are important at short range and, as demonstrated by the Faraday cage screening effect, can also be critical at intermediate to long range. However, at the short-range distances typical of vdW equilibria, many-body effects often appear to cancel, allowing the combination of GGAs with pairwise-additive schemes like D3 to become widely successful $(2,31,32,78)$ (Table 1$)$. Nevertheless, Table 1 indicates that replacing pairwise-additive methods like D3 with many-body ones like MBD allows all Dobson-B effects to be included at short to intermediate range, often leading to improved descriptions at vdW contact $(4,24,35,37-45)$. One would hope that such improvements continued down to the very short length scales of chemical bonding and beyond.

In Fig. 3, properties of the perceived dispersion interaction at short to intermediate separations are expanded from inside vdW contact $\left(d \sim d_{\mathrm{e}}=6.65 \AA\right.$ ) to the very short distances typical of covalent and ionic bonding ( $d \sim 4 \AA$ if alignment allows) down toward the unified atom limit at which nuclear fusion would occur $(d \rightarrow 0)$. The MBD-FI screening curve follows the $1 / d$ law well until near close contact $(\sim 9 \AA)$, undergoing some wiggles down to chemical bonding distances $(5 \AA)$ and then going flat (Fig. 3C). In contrast, the D3 and B3(ABC) screening is near unity until $\sim 5 \AA$ and then behaves very erratically. This arises as the London $C_{6}$ coefficients are coordination dependent, allowing implicit inclusion of Dobson-B effects. However, the implementation of this concept in D3 and D3(ABC) is purely empirical. It is intended to describe typical chemical bonding scenarios, but simply compressing the BGB system together as is done in Fig. 3 does not correspond to any known chemical process and hence is not something that these methods were designed to do, leading to the erratic results. Our focus here is not in these troubles with the more empirical approaches, but rather the differences between them and MBD-FI in the region below vdW contact heading toward covalent bonding for which the D3 and D3(ABC) descriptions may be thought to be realistic: MBD-FI predicts strong antiscreening, an effect that flows from its dramatically improved description at intermediate distances exemplified by Faraday cage screening and other effects (46), while D3 and D3(ABC) do not.

A design criterion for D3 was that, using a predefined algorithm, it can be automatically applied to any density functional, as it has been done to at least 115 different density functionals $(30,31,33)$. How the vdW correction links to the underlying description of electron correlation embodied in each functional has been a focus of much of the empiricism of the method. Significantly, it can also be applied to HF, to which it adds a description of the short-range electron correlation that can be competitive with descriptions provided by GGA-type density functionals such as Perdew-Burke-Ernzerhof (PBE) (30, 69, 79,
80). The central idea here is one of compatibility: The expectation is that adding D3 to any density functional largely just improves that functional's description of covalent bonding.

In comparison, the development of MBD-based approaches are just in their infancy. MBD has been applied now to eight density functionals (45). Like D3, MBD assumes an empirical damping function specifying the changeover between the covalent and dispersive bonding regimes. However, for MBD, its form and parameter are chosen considering only data pertinent to dispersive interactions (45).

To bring focus to this discussion, we note that recently it was found that vdW forces can control the internal chemical structure of $\mathrm{CuInP}_{2} \mathrm{~S}_{6}$ and $\mathrm{CuBiP}_{2} \mathrm{Se}_{6}$ monolayers (12), that specific $\mathrm{Cu}-\mathrm{Cu}$ vdW interactions outcompete ionic forces to control antiferroelectric/ferroelectric arrangements of stacked monolayers (13), and that the nature of the vdW forces controlling stacking is uncorrelated with the nature of the vdW forces controlling internal chemical structure (12). Furthermore, D3-based approaches give results for the internal chemical structure that closely relate to experiment, being much improved on compared with the underlying density functional used alone, whereas MBD performs very poorly in this regard, degrading predictions.

In Fig. $3 D$, the total dispersion energy (energy for method including dispersion less energy using only the underlying density functional) is shown for the BGB trilayer system. At short to intermediate distances in which Faraday cage screening is important, D3 and D3(ABC) overestimate the vdW attraction compared with MBD-FI. However, this situation reverses at very short distances comparable to covalent bond lengths with $d<5 \AA$. At short to intermediate distances, the D3 and D3(ABC) total dispersion energies scale as expected (21) as $d^{-4}$, with MBD-FI scaling similarly as $d^{-3.8}$. However, at extremely short distances with $d<4 \AA$, the MBD-FI scaling reducing to $d^{-0.94}$, but the D3 and $\mathrm{D} 3(\mathrm{ABC})$ interactions are being even more greatly dampened. This dampening of D3 dispersion energies compared with the continuing increase of MBD-type energies is a general effect, highlighted in the Inset in the figure, which shows the total dispersion energy for the $\mathrm{H}_{2}$ molecule from intermediate distances through vdW contact and then covalent contact to the unified atom limit. The vdW force must approach a constant value in the unified-atom limit (81), as exemplified only by D3.

\section{Discussion}

Our results show that the dramatic switching off of the vdW force demonstrated by the experiments of Tsoi et al. (20) is explained by Faraday cage-type screening effects involving the many-body response of all atoms in graphene to spontaneous quantum fluctuations in neighboring matter (Dobson-B effects). Modern computational methods can quantitatively reproduce these effects, provided that they include all responses to the fluctuations, as does MBD. However, subtleties, including correct asymptotic limits, needed to establish full quantitative agreement may require metallic (Dobson-C) models (68), this becoming critical whenever fully metallic low-dimensional systems are involved (21). Extension to include very long-range effects $(17,21)$ may also be required.

Methods like D3(BJ,ABC) that only partially include these responses are found to be inadequate for a general description of $\mathrm{vdW}$ screening in layered systems. These inadequacies are profound when screened silica bilayers are separated at distances of order twice the vdW separation and scale up with $d^{-1}$ at larger (but still intermediate) distances. However, the screening effect cancels as the equilibrium position is reached, allowing simplistic pairwise-additive methods to often yield good results for this important class of problems (2, 29-33, 56-60).

We see here that the Tsoi et al. (20) experiment reveals fundamental physics critical to the understanding of systems at equilibrium as neglected screening (and other Dobson-B) effects 
appear as strong functions of separation around equilibrium structures. To better understand the dispersion force from asymptotic distances down to contact, there is great need for this experiment to be repeated so as to determine accurately the screening ratio $\Delta E_{s} / \Delta E_{u}$ as a function of distance $d$.

In terms of the conceptual understanding of the vdW force and its links to chemical forces, the results provide an understanding as to how Dobson-B terms behave at very short length scales below vdW equilibrium separations and their link with covalent bonding. Poor results found recently from MBD calculations for the competition between covalent and vdW forces controlling $\mathrm{CuInP}_{2} \mathrm{~S}_{6}$ and $\mathrm{CuBiP}_{2} \mathrm{Se}_{6}$ monolayers $(12,13)$, poor results demonstrated for the dispersion force perceived in the $\mathrm{H}_{2}$ molecule in the unified-atom limit, may be related. These results therefore provide insights into how one might improve vdW models at atomic bond-length scales via simultaneous improvements to Dobson-A and -B effects, tailoring atomscaled vdW forces. Clearly, modification of the design and parameterization of MBD approaches (45) to include these effects is highly desired.

More fundamentally, the results presented illustrate the competitive (and hard-to-define) role played by Dobson-A and -B terms at atomic scales; for example, the effect of the horizontal field lines in the right diagram of Fig. 2 can by mimicked by local changes to the response function. Can methods be developed that seamlessly include Faraday cage screening and covalent bonding? To make progress on this issue, an analytical understanding of how the RPA perceives Dobson-B effects at short range is urgently required. We feel that a numerical RPA correlation energy calculation at all separations, if feasible for the small energy differences investigated here, would be close to the current state of the art and would also very illuminating for these problems. Simplified versions of RPA (64-67) may help here.

In terms of nanotechnological applications, the consequences of Faraday cage screening may become significant in future devices made from $2 \mathrm{D}$ materials. Already, multiple layered systems are being made, allowing screening to occur (1). However, the screening effect acts to modify long-range interactions that are themselves small, so its influence may not be competitive with other effects in asserting control of self-assembly and device structure. This is in contrast to possible profound screening effects that conducting layers could have on functional properties housed inside layers that they separate, acting like a "ground plane" in electronics circuit boards.

To make the effects of vdW screening competitive, the objects being screened from each other need to themselves be large. The silica substrate and AFM tip used in the Tsoi et al. (20) experiment are examples of this effect. In this way, a conducting intermediary layer could dramatically reduce the force between two nanostructures of considerable thickness. If some external signal (e.g., transmitted current, applied electric or magnetic

1. Geim AK, Grigorieva IV (2013) van der Waals heterostructures. Nature 499 419-425.

2. Reimers JR, et al. (2015) A priori calculations of the free energy of formation from solution of polymorphic self-assembled monolayers. Proc Natl Acad Sci USA 112 E6101-E6110.

3. Batista CAS, Larson RG, Kotov NA (2015) Nonadditivity of nanoparticle interactions Science 350:1242477.

4. Ambrosetti A, Ferri N, DiStasio RA, Jr, Tkatchenko A (2016) Wavelike charge density fluctuations and van der Waals interactions at the nanoscale. Science 351:1171-1176.

5. Bilić A, Reimers JR, Hush NS, Hoft RC, Ford MJ (2006) Adsorption of benzene on copper, silver, and gold surfaces. J Chem Theory Comput 2:1093-1105.

6. Schreiner PR, et al. (2011) Overcoming lability of extremely long alkane carboncarbon bonds through dispersion forces. Nature 477:308-311.

7. Nerngchamnong N, et al. (2013) The role of van der Waals forces in the performance of molecular diodes. Nat Nanotechnol 8:113-118.

8. Das PK, Samanta S, McQuarters AB, Lehnert N, Dey A (2016) Valence tautomerism in synthetic models of cytochrome P450. Proc Natl Acad Sci USA 113:6611-6616. field, or an optical signal) could switch the conductance on or off, then the friction force between the nanoscale materials would also be modulated, blocking or facilitating slippage or separation.

The vdW force is not traditionally viewed as being controllable, as ionic forces, hydrogen bonding, and covalent bonding are. We now know that it can compete with these traditional chemical motifs to control structure and function (11). Developing the means of controlling and manipulating it therefore opens up new ways for designing functional materials and devices. The screening reported here shows that such manipulation is not only possible but can be modeled using existing theories, thereby enabling computer-led design of such materials.

\section{Materials and Methods}

We place all structures in a hexagonal periodic box with dimensions of $5.17 \times$ $5.17 \AA$ in the bilayer plane and $50 \AA$ long, as indicated in Fig. 1. Initial density functional calculations are then performed using full 3D periodicity, as the long box length results in negligible PBE interactions between silica bilayer sheets. For the subsequent vdW dispersion corrections, however, only 2D periodic imaging is performed as these interactions remain significant on the 50 - $\AA$ scale.

All calculations are performed by $\operatorname{VASP}(71,72)$ using $24 \times 24 \times 1 k$-points. The PBE density functional (73) is used along with PAW pseudopotentials describing core electrons (74). A plane-wave basis set is used set to an energy cutoff of $500 \mathrm{eV}$. The shortest-wavelength plane wave used in the direction perpendicular to the bilayers is $0.1 \AA$, and all calculated structures have layers translated by integral multiples of it. This ensures that the relationship between the grid points used in the electronic structure calculations and the precise nuclear coordinates remains invariant, seriously increasing the precision to which the calculations are performed.

In calculations of this type, the graphene lattice must be commensurate with that used for the silica bilayers. The two lattices are of the same type, but the PBE-calculated lattice constant is $5.18 \AA$ for silica bilayers and $4.92 \AA$ for graphene. In the calculations, a compromise value of $5.17 \AA$ is used. The calculated band structures for the silica bilayer and for graphene at this value closely mimic those at the equilibrium geometries, manifesting the silica bilayer as a high bandgap insulator, as well as the graphene Dirac cones.

Dispersion correction options available in VASP allow for variations of the D3 and MBD methods. In all cases, we use PBE as the base functional. For D3, we select the methods known as "D3(BJ)" (VASP option "IVDW =12") and "D3(BJ,ABC)" (VASP option "IVDW = 12" and "noabc = .false.") involving Becke-Johnson damping (both) and three-body Axilrod-Teller corrections (the latter only) $(29,69)$. For $\operatorname{MBD}(41,70)$, we employ the FI method $(43)$, which incorporates a superior polarizability model for oxides and uses eigenvalue rescaling to avoid the polarization catastrophe that can occur in highly polarizable systems (VASP option "IVDW $=263$ ") $(42,75,82)$. Also, to enhance precision for the MBD calculations, we use large summation grid (VASP option "VDW_MBD_SIZE = 88 1"), which can fully capture the longrange screening.

ACKNOWLEDGMENTS. We thank the National Natural Science Foundation of China for support through Grant 11674212.

9. Wagner JP, Schreiner PR (2016) London dispersion decisively contributes to the thermodynamic stability of bulky NHC-coordinated main group compounds. J Chem Theory Comput 12:231-237.

10. Reimers JR, Ford MJ, Halder A, Ulstrup J, Hush NS (2016) Gold surfaces and nanoparticles are protected by $\mathrm{Au}(0)$-thiyl species and are destroyed when $\mathrm{Au}(\mathrm{I})$-thiolates form. Proc Natl Acad Sci USA 113:E1424-E1433.

11. Reimers JR, Ford MJ, Marcuccio SM, Ulstrup J, Hush NS (2017) Competition of van der Waals and chemical forces on gold-sulfur surfaces and nanoparticles. Nat Rev Chem 1: 0017.

12. Tawfik SA, Reimers JR, Stampfl C, Ford MJ (2018) van der Waals forces control the internal chemical structure of monolayers within the lamellar materials $\mathrm{CulnP}_{2} \mathrm{~S}_{6}$ and $\mathrm{CuBiP}_{2} \mathrm{Se}_{6}$. J Phys Chem C 122:22225-22802.

13. Reimers JR, Tawfik SA, Ford MJ (September 17, 2018) Van der Waals forces control ferroelectric-antiferroelectric ordering in $\mathrm{CuInP}_{2} \mathrm{~S}_{6}$ and $\mathrm{CuBiP}_{2} \mathrm{Se}_{6}$ laminar materials. Chem Sci, 10.1039/C1038SC01274A.

14. Chen L, et al. (2017) lon sieving in graphene oxide membranes via cationic control of interlayer spacing. Nature 550:380-383. 
15. Shi G, et al. (2018) Two-dimensional Na-Cl crystals of unconventional stoichiometries on graphene surface from dilute solution at ambient conditions. Nat Chem 10 776-779.

16. Widmer DR, Schwartz BJ (2018) Solvents can control solute molecular identity. Nat Chem 10:910-916.

17. Ambrosetti A, Silvestrelli PL (2018) Hidden by graphene-towards effective screening of interface van der Waals interactions via monolayer coating. Carbon 139:486-491.

18. Woods LM, et al. (2016) Materials perspective on Casimir and van der Waals interactions. Rev Mod Phys 88:045003.

19. Rodriguez-Lopez P, Kort-Kamp WJM, Dalvit DAR, Woods LM (2017) Casimir force phase transitions in the graphene family. Nat Commun 8:14699.

20. Tsoi $S$, et al. (2014) van der Waals screening by single-layer graphene and molybdenum disulfide. ACS Nano 8:12410-12417.

21. Dobson JF (2014) Beyond pairwise additivity in London dispersion interactions. Int J Quantum Chem 114:1157-1161.

22. Lebègue $\mathrm{S}$, et al. (2010) Cohesive properties and asymptotics of the dispersion interaction in graphite by the random phase approximation. Phys Rev Lett 105:196401.

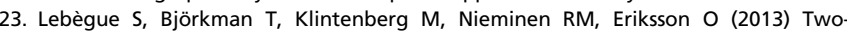
dimensional materials from data filtering and ab initio calculations. Phys $\operatorname{Rev} \times 3$ 031002.

24. Gould T, Lebègue S, Björkman T, Dobson JF (2016) 2D structures beyond graphene: The Brave New World of layered materials and how computers can help discover them. Semiconductors and Semimetals, eds lacopi F, Boeckl JJ, Jagadish C (Elsevier, Amsterdam), Vol 95, Chap 1, pp 1-33.

25. Reimers JR, Li M, Wan D, Gould T, Ford MJ (2017) Surface adhesion. Noncovalent Interactions in Quantum Chemistry and Physics: Theory and Applications, eds de la Roza AO, DiLabio GA (Elsevier, Amsterdam), Chap 13.

26. Dobson JF, White A, Rubio A (2006) Asymptotics of the dispersion interaction: Analytic benchmarks for van der Waals energy functionals. Phys Rev Lett 96:073201.

27. Dobson JF, Gould T, Vignale G (2014) How many-body effects modify the van der waals interaction between graphene sheets. Phys Rev X 4:021040.

28. Dobson JF, Savin A, Ángyán JG, Liu R-F (2016) Spooky correlations and unusual van der Waals forces between gapless and near-gapless molecules. J Chem Phys 145 204107

29. Grimme S, Antony J, Ehrlich S, Krieg H (2010) A consistent and accurate ab initio parametrization of density functional dispersion correction (DFT-D) for the 94 elements H-Pu. J Chem Phys 132:154104.

30. Goerigk L, Grimme S (2011) A thorough benchmark of density functional methods for general main group thermochemistry, kinetics, and noncovalent interactions. Phys Chem Chem Phys 13:6670-6688

31. Goerigk L, et al. (2017) A look at the density functional theory zoo with the advanced GMTKN55 database for general main group thermochemistry, kinetics and noncovalent interactions. Phys Chem Chem Phys 19:32184-32215.

32. Goerigk L (2017) A comprehensive review of the DFT-D3 London-dispersion method. Noncovalent Interactions in Quantum Chemistry and Physics: Theory and Applications, eds Otero de la Roza A, DiLabio G (Elsevier, Amsterdam), pp 195-219.

33. Mehta N, Casanova-Páez M, Goerigk L (2018) Semi-empirical or non-empirical doublehybrid density functionals: Which are more robust? Phys Chem Chem Phys 20: 23175-23194.

34. Reimers JR, Ford MJ, Marcuccio SM, Ulstrup J, Hush NS (2017) Erratum: Competition of van der Waals and chemical forces on gold-sulfur surfaces and nanoparticles. Nat Rev Chem 1:0034.

35. Tkatchenko A, DiStasio RA, Car R, Scheffler M (2012) Accurate and efficient method for many-body van der Waals interactions. Phys Rev Lett 108:236402.

36. Gobre VV, Tkatchenko A (2013) Scaling laws for van der Waals interactions in nanostructured materials. Nat Commun 4:2341.

37. Ruiz VG, Liu W, Tkatchenko A (2016) Density-functional theory with screened van der Waals interactions applied to atomic and molecular adsorbates on close-packed and non-close-packed surfaces. Phys Rev B 93:035118.

38. Tkatchenko A (2015) Current understanding of Van der Waals effects in realistic materials. Adv Funct Mater 25:2054-2061.

39. Reilly AM, Tkatchenko A (2015) van der Waals dispersion interactions in molecular materials: Beyond pairwise additivity. Chem Sci (Camb) 6:3289-3301.

40. Maurer RJ, Ruiz VG, Tkatchenko A (2015) Many-body dispersion effects in the binding of adsorbates on metal surfaces. J Chem Phys 143:102808.

41. Ambrosetti A, Reilly AM, Distasio RA, Jr, Tkatchenko A (2014) Long-range correlation energy calculated from coupled atomic response functions. J Chem Phys 140:18A508.

42. Bučko T, Lebègue $S$, Gould T, Ángyán JG (2016) Many-body dispersion corrections for periodic systems: An efficient reciprocal space implementation. J Phys Condens Matter 28:045201.

43. Gould T, Lebègue S, Ángyán JG, Bučko T (2016) A fractionally ionic approach to polarizability and van der Waals many-body dispersion calculations. I Chem Theory Comput 12:5920-5930.

44. Hermann J, DiStasio RA, Jr, Tkatchenko A (2017) First-principles models for van der Waals interactions in molecules and materials: Concepts, theory, and applications. Chem Rev 117:4714-4758.

45. Hermann J, Tkatchenko A (2018) Electronic exchange and correlation in van der Waals systems: Balancing semilocal and nonlocal energy contributions. J Chem Theory Comput 14:1361-1369.

46. Gould T, Johnson ER, Tawfik SA (2018) Are dispersion corrections accurate outside equilibrium? A case study on benzene. Beilstein J Org Chem 14:1181-1191.
47. Lifshitz EM (1956) The theory of molecular attractive forces between solids. J Exp Theor Phys 29:94-110.

48. Dzyaloshinskii IE, Lifshitz EM, Lev PP (1961) General theory of van der Waals' forces. Sov Phys Usp 4:153.

49. Eisenschitz R, London F (1930) Über das Verhältnis der van der Waalsschen Kräfte zu den Homöopolaren Bindungskräften. Z Phys 60:491-527.

50. White A, Dobson JF (2008) Enhanced dispersion interaction between quasi-onedimensional conducting collinear structures. Phys Rev B Condens Matter Mater Phys 77:075436.

51. Liu RF, Angyan JG, Dobson JF (2011) Dispersion interaction in hydrogen-chain models. J Chem Phys 134:114106

52. Zou J, et al. (2013) Casimir forces on a silicon micromechanical chip. Nat Commun 4: 1845

53. Dobson JF, et al. (2002) Prediction of dispersion forces: is there a problem? Aust $J$ Chem 54:513-527.

54. Møller C, Plesset MS (1934) Note on an approximation treatment for many-electron systems. Phys Rev A 46:618-622.

55. Purvis GP, III, Bartlett RJ (1982) A full coupled-cluster singles and doubles model: The inclusion of disconnected triples. J Chem Phys 76:1910-1918.

56. Christian MS, Otero-de-la-Roza A, Johnson ER (2016) Surface adsorption from the exchange-hole dipole moment dispersion model. J Chem Theory Comput 12: 3305-3315.

57. Dion M, Rydberg H, Schröder E, Langreth DC, Lundqvist BI (2004) van der Waals density functional for general geometries. Phys Rev Lett 92:246401.

58. Berland K, et al. (2015) van der Waals forces in density functional theory: A review of the vdW-DF method. Rep Prog Phys 78:066501.

59. Lee K, Murray ÉD, Kong L, Lundqvist BI, Langreth DC (2010) Higher-accuracy van der Waals density functional. Phys Rev B Condens Matter Mater Phys 82:081101.

60. Peng $H$, Yang Z-H, Perdew JP, Sun J (2016) Versatile van der Waals density functional based on a meta-generalized gradient approximation. Phys $\operatorname{Rev} X$ 6:041005.

61. Gould T, Dobson JF, Lebègue $S$ (2013) Effects of a finite Dirac cone on the dispersion properties of graphite. Phys Rev B Condens Matter Mater Phys 87:165422.

62. Eshuis H, Bates JE, Furche F (2012) Electron correlation methods based on the random phase approximation. Theor Chem Acc 131:1084.

63. Dobson JF, Gould T (2012) Calculation of dispersion energies. J Phys Condens Matter 24:073201.

64. Bates M, Lunt RR (2017) Organic salt photovoltaics. Sustainable Energy Fuels 1: 955-968.

65. Erhard J, Bleiziffer P, Görling A (2016) Power series approximation for the correlation kernel leading to Kohn-Sham methods combining accuracy, computational efficiency, and general applicability. Phys Rev Lett 117:143002.

66. Kaltak M, Klimeš J, Kresse G (2014) Cubic scaling algorithm for the random phase approximation: Self-interstitials and vacancies in Si. Phys Rev B Condens Matter Mater Phys 90:054115.

67. Kaltak M, Klimeš J, Kresse G (2014) Low scaling algorithms for the random phase approximation: Imaginary time and laplace transformations. J Chem Theory Comput 10:2498-2507.

68. Dobson JF, Gould T, Lebègue S (2016) Layer response theory: Energetics of layered materials from semianalytic high-level theory. Phys $\operatorname{Rev} B$ 93:165436.

69. Grimme S, Ehrlich S, Goerigk L (2011) Effect of the damping function in dispersion corrected density functional theory. J Comput Chem 32:1456-1465.

70. Bučko T, Lebègue S, Hafner J, Ángyán JG (2013) Improved density dependent correction for the description of London dispersion forces. J Chem Theory Comput 9 : 4293-4299.

71. Kresse G, Hafner J (1993) Ab initio molecular dynamics for liquid metals. Phys Rev $B$ Condens Matter 47:558-561.

72. Kresse G, Furthmüller J (1996) Efficiency of ab-initio total energy calculations for metals and semiconductors using a plane-wave basis set. Comput Mater Sci 6:15-50.

73. Perdew JP, Burke K, Ernzerhof M (1996) Generalized gradient approximation made simple. Phys Rev Lett 77:3865-3868.

74. Kresse G, Joubert D (1999) From ultrasoft pseudopotentials to the projecto augmented-wave method. Phys Rev B Condens Matter Mater Phys 59:1758-1775.

75. Larmier K, Tada S, Comas-Vives A, Copéret C (2016) Surface sites in Cu-nanoparticles: Chemical reactivity or microscopy? J Phys Chem Lett 7:3259-3263.

76. Włodarczyk R, et al. (2012) Tuning the electronic structure of ultrathin crystalline silica films on Ru(0001). Phys Rev B Condens Matter Mater Phys 85:85403.

77. Björkman T, Gulans A, Krasheninnikov AV, Nieminen RM (2012) Are we van der Waals ready? J Phys Condens Matter 24:424218.

78. Otero de la Roza A, DiLabio G, eds (2017) Noncovalent Interactions in Quantum Chemistry and Physics: Theory and Applications (Elsevier, Amsterdam).

79. Brandenburg JG, Grimme S (2014) Dispersion corrected Hartree-Fock and density functional theory for organic crystal structure prediction. Top Curr Chem 345:1-23.

80. Goerigk L, Collyer CA, Reimers JR (2014) Recommending Hartree-Fock theory with London-dispersion and basis-set-superposition corrections for the optimization or quantum refinement of protein structures. J Phys Chem B 118:14612-14626.

81. Koide A (1976) A new expansion for dispersion forces and its application. J Phys $B$ 3173-3183.

82. Kim WJ, Kim M, Lee EK, Lebègue $\mathrm{S}$, Kim H (2016) Failure of density functional dispersion correction in metallic systems and its possible solution using a modified manybody dispersion correction. J Phys Chem Lett 7:3278-3283. 